\title{
Restoration of F508-del Function by Transcomplementation: The Partners Meet in the Endoplasmic Reticulum
}

\author{
Emily Anne Smith Bergbower Inna Sabirzhanova Clément Boinot \\ William B. Guggino Liudmila Cebotaru \\ Departments of Medicine and Physiology, Johns Hopkins University, Baltimore, MD, USA
}

\section{Key Words}

Gene therapy • Cystic fibrosis • Bimolecular interaction - Transcomplementation - Truncated CFTR

\begin{abstract}
Background/Aims: Because of the small size of adeno-associated virus, AAV, the cystic fibrosis conductance regulator, CFTR, CDNA is too large to fit within AAV and must be truncated. We report here on two truncated versions of CFTR, which, when inserted into AAV1 and used to infect airway cells, rescue F508-del CFTR via transcomplementation. The purpose of this study is to shed light on where in the cell transcomplementation occurs and how it results in close association between the endogenous F508-del and truncated CFTR. Methods: We treated CF airway cells (CFBE410) with AAV2/1 (AAV2 inverted terminal repeats/AAV1 capsid) containing truncated forms of CFTR, $\triangle 264$ and $\triangle 27-264$ CFTR, who can restore the function of F508-del by transcomplementation. We addressed the aims of the study using a combination of confocal microscopy and short circuit currents measurements. For the latter, CF bronchial epithelial cells (CFBE) were grown on permeable supports. Results: We show that both F508del and the truncation mutants colocalize in the ER and that both the rescued F508-del and the transcomplementing mutants reach the plasma membrane together. There was significant fluorescence resonance energy transfer (FRET) between F508-del and the transcomplementing mutants within the endoplasmic reticulum (ER), suggesting that transcomplementation occurs through a bimolecular interaction. We found that transcomplementation could increase the Isc in CFBE410 cells stably expressing additional wt-CFTR or F508-del and in parental CFBE410 cells expressing endogenous levels of F508-del. Conclusion: We conclude that the functional rescue of F508-del by transcomplementation occurs via a bimolecular interaction that most likely begins in the ER and continues at the plasma membrane. These results come at an opportune time for developing a gene therapy for CF and offer new treatment options for a wide range of CF patients.




\section{Cellular Physiology Cell Physiol Biochem 2019;52:1267-1279 \\ \begin{tabular}{ll|l} 
and Biochemisty $10.33594 / 000000089$ & (c) 2019 The Author(s). Published by \\
Cell Physiol Biochem Press GmbH\&Co. KG
\end{tabular} \\ Smith Bergbower et al.: Restoring Function of F508-del by Transcomplementation}

\section{Introduction}

Cystic fibrosis (CF), an autosomal disorder most common in Caucasians, is caused by defects in the CFTR, a chloride channel found in mucosal epithelia [1]. The most common mutant, F508-del, is well known to be retained within the ER and degraded by proteasomes [2]. There are well over 1000 mutations in the CFTR gene that cause a myriad of problems, including premature degradation, aberrant channel conductance or gating, premature translation termination resulting from early stop codons, and aberrant splicing [3, 4].

Gene therapy: Shortly after the CF gene was identified, there was tremendous enthusiasm for gene therapy as the ultimate way to restore health to CF patients [5]. This enthusiasm waned as the challenges in achieving a gene therapy became evident. One vector that shows promise for CF gene therapy is adeno-associated virus (AAV). AAV2 gene transfer has been tested extensively in humans $[6,7]$ and found to be safe. Early studies using the first generation of virus, AAV2-CFTR, demonstrated gene transfer and transduction, but a largescale repeat-dosing study with over 100 patients did not. There were two major reasons for this lack of clinical efficacy: First, AAV2 was not very efficient in transducing lung cells [8]; second, the weak promoter in the vector construct did not express enough protein to be effective [9]. To overcome the first hurdle, we developed CFTR viral vectors based on the AAV1 serotype [10]. The solution to the second problem was to add a more powerful chicken $\beta$-actin (CBA) [11] or tg38 [12] promoter to boost gene expression. However, the addition of the promoter required truncating CFTR in order to fit it within the packaging capacity of AAV $[13,14]$.

Transcomplementation of F508-del. In order to fit CFTR within the packaging capacity of AAV, we created the truncation mutant, $\triangle 264$ CFTR, by removing the first four transmembrane-spanning domains of CFTR. Because $\Delta 264$ CFTR had low protein expression [15], we created another truncation mutant, $\Delta 27-264$ CFTR [16], to improve the levels of steady-state protein expressed by the original truncation mutant. To create this new construct, we added the first 26 amino acids of wild type (wt) CFTR onto the N-terminus of our $\Delta 264$ CFTR. We based the design of $\Delta 27-264$ CFTR on our prior knowledge that the first 26 amino acids of CFTR are important for enhanced protein expression [17]. Others have demonstrated that the N-terminal tail of CFTR binds to important trafficking molecules such as syntaxin 1a [18]. Indeed, we showed that $\Delta 27-264$ CFTR expresses higher levels of steady-state protein than does $\Delta 264$ CFTR [15].

When we used it to infect monkey lungs, AAV5- 264 CFTR increased the levels of endogenous wt CFTR protein [19]. In co-transfection studies in Cos7 and CFBE410 cells and in the transfection of $\Delta 264$ CFTR into cells stably expressing wt and F508-del-CFTR, $\Delta 264$ CFTR increased the wt CFTR protein level and the ratio of mature $C$ bands to immature $B$ bands [15]. We discovered that the truncation mutants were not functioning on their own, but were rescuing F508-del by transcomplementation [16]. Transcomplementation occurs when certain truncation mutants such as $\Delta 264$ CFTR and $\Delta 27-264$ CFTR bind to F508-del and rescue its function. Rescue of $\Delta$ F508-CFTR by transcomplementation has been observed previously $[20,21]$. One common theme among all these studies is that truncation mutants of CFTR capable of transcomplementation are extremely efficiently degraded. Thus, one theory holds that transcomplementation rescues $\triangle$ F508-CFTR via chaperone competition or displacement: Certain CFTR truncation mutants avidly bind to ERAD proteins such as valosin-containing protein (VCP), ATPase homolog 1 (Aha1), and histone deacetylase 6 (HDAC6) [21]. As a result, these mutants compete for binding or displace F508-del from the ERAD proteins, thereby allowing F508-del to reach the cell surface. A second theory holds that other truncation mutants bind directly to F508-del and effect its rescue through domain interactions between the different CFTR molecules. Thus, chaperone displacement would be a consequence of a structurally rescued F508-del. The latter alternative would suggest that the transcomplementing truncation mutants bind to F508-del during translation, stabilize the protein, and remain together with F508-del as it is processed to the plasma membrane. Because this scenario has not be demonstrated experimentally, the goal of the present study 


\section{Cellular Physiology Cell Physiol Biochem 2019;52:1267-1279 \\ \begin{tabular}{l|l}
\hline DOI: 10.33594/000000089 & ( 2019 The Author(s). Published by
\end{tabular} \\ Published online: 27 April $2019 \quad$ Cell Physiol Biochem Press GmbH\&Co. KG

was use confocal microscopy to determine where F508-del and the transcomplementing truncation mutants actually associate within the cell and whether the transcomplementing truncation mutants and F508-del reside at the plasma membrane.

\section{Materials and Methods}

\section{Cell culture}

CFBE410 cells stably transfected with F508-del (a gift of Erik Sorscher) or with wt CFTR, as well as parental cells (no additional CFTR, a gift of Dieter Gruenert [22]) were maintained in minimal essential Eagle's medium (MEM, Invitrogen) with 10\% FBS, penicillin/streptomycin, L-glutamate (200 mM), and puromycin (5 $\mu \mathrm{g} / \mathrm{mL}$, Sigma).

\section{Microscopy}

A Zeiss LSM 510 laser scanning system and 63x oil-immersion lens were used. CFBE410 parental cells were seeded onto cover glasses and later transiently transfected with the plasmids KDEL-td-tomato, F508-del CFTR-GFP, $\Delta 27-264$ CFTR-mCherry, and $\Delta 264$ CFTR-mCherry for $48 \mathrm{~h}$ using Lipofectamine 2000. All these constructs contain the CBA promoter [13]. All tags were placed on the C-terminal domain. The following steps were done at room temperature to prepare slides for assessment: After two washes with cold DPBS, the cells were fixed with $4 \%$ paraformaldehyde for $15 \mathrm{~min}$. They were then permeabilized with 0.3\% Triton X-100 in DPBS for 5-7 min and blocked with 3\% bovine albumin serum (BSA) for 45 min. After blocking, cells were washed once with DPBS and then incubated with primary antibodies in 3\% BSA for 1 h. The primary antibodies used were: anti-KDEL (Enzo Life Sciences \#ADI-SPA-827-D), anti-golgin-97 (Life Technologies \#A-21270), and anti-pan cadherin (Abcam \#ab6528). CFTR was detected by its fluorescent tags or with anti-CFTR antibody 596 [23]. After incubation with primary antibody, the cells were incubated with secondary antibody: goat anti-mouse Alexa 647, goat anti-mouse AlexaFluor 488, or goat anti-mouse AlexaFluor 594. The cells were incubated with a 1:1000 dilution of DAPI for $5 \mathrm{~min}$ and washed three times with DPBS, then mounted using ProLong Gold Antifade (Invitrogen). Extended-focus images were taken on the Zeiss LSM 510 and processed for export as TIF files using Imaris Imaging Software. We verified previously that transcomplementation-tagged versions of CFTR undergo transcomplementation in a manner similar to that of those without tags [15].

FRET

$\triangle 264 C F T R$ was subcloned from pcDNA3.1 into pmCherry-C1 (Clontech, \#632524) using the following primers: 5'-tagggtaccatgatcgagaacatccaatctgtta-3' and 5'- taaacgggccctaaagccttgtatcttgca-3'. $\Delta 27-264$ CFTR was subcloned from pcDNA3.1 into pmCherry-C1 (Clontech, \#632524), and $\triangle$ F508-CFTR was subcloned from pcDNA3.1 into pAcGFP1-C1 (Clontech, \#632470) using the following primers: 5'-ctaggtaccatgcagaggtcgcctctggaaaat-3' and 5'-taaacgggccctaaagccttgtatcttgca-3'. The GFP/mCherry (donor/acceptor) pair was used for acceptor photobleach FRET. Images for GFP and mCherry were collected before and after photobleaching with the 594-nm argon/2 laser at the maximum intensity. From the images, the signal intensity of GFP was measured with Zeiss image software, and FRET efficiency was calculated using the following formula:

$$
\text { FRET efficiecy }=\left(\frac{\mathrm{I}_{\mathrm{Past}}-\mathrm{I}_{\mathrm{PTe}}}{\mathrm{I}_{\mathrm{PTe}}}\right)
$$

where $\mathrm{I}_{\text {Pre }}$ and $\mathrm{I}_{\text {Post }}$ correspond to the GFP signal intensity (with background signal subtracted) before and after photobleach, respectively. Note that FRET between $\Delta 27-264$ and $\Delta$ F508 does occur within the ER region.

\section{Short-circuit currents}

Parental cells or CFBE410 cells stably expressing $\triangle$ F508-CFTR or wt CFTR were grown on Transwell supports and treated with $10 \mu \mathrm{l}$ of AAV2/1 (AAV1capsid/AAV2 ITR) containing either $\Delta 264$ CFTR $\left(3.75 X 10^{12}\right.$ vector genomes $[\mathrm{vg}] / \mathrm{ml})$ or $\Delta 27-264$ CFTR $\left(2.19 \times 10^{12} \mathrm{vg} / \mathrm{ml}\right)$. Virus was produced at the University of Florida Vector Core Facility. The short-circuit currents (Isc) were measured in Ussing-type chambers (Physiological Instruments; San Diego, CA). Confluent CF bronchial epithelial cells (CFBE41o') stably expressing 


\section{Cellular Physiology Cell Physiol Biochem 2019;52:1267-1279 \\ \begin{tabular}{ll|l} 
and Biochemistry $10.33594 / 000000089$ & $\begin{array}{l}\text { DO } 2019 \text { The Author(s). Published by } \\
\text { Published online: } 27 \text { April } 2019\end{array}$ & Cell Physiol Biochem Press GmbH\&Co. KG \\
\cline { 2 - 3 }
\end{tabular}

F508-del or wt CFTR were seeded onto $12 \mathrm{~mm}$-diameter Costar $\AA$ Snapwell ${ }^{\mathrm{TM}}$ cell culture inserts (Corning Costar, Acton, MA; 3801) and cultured for 7 days to establish polarized monolayers. Once the monolayers had reached the relevant resistance, the inserts were mounted in an Ussing-type chamber and bathed in one of the solutions described below, maintained at $37^{\circ} \mathrm{C}$, and stirred by bubbling with room air.

Short-circuit currents (Isc) were measured by voltage-clamping the transepithelial voltage across the monolayers to $0 \mathrm{mV}$ with a multichannel voltage-current clamp amplifier (model VCC MC6, Physiologic Instruments) as described previously [24].

\section{Results}

F508-del and the N-terminal truncation mutants are located within the ER

Although transcomplementation is postulated to occur via a bimolecular interaction [25], the location of the transcomplementing forms of CFTR in the cell is unknown. In order to study their location, we utilized confocal microscopy. Fig. 1 shows that F508-del is located within a perinuclear compartment. F508-del localized with KDEL, a marker for the ER [26], confirming what is already well known: that F508-del is a mutant protein whose trafficking is arrested in the ER [27]. However, as we have observed previously [15], a small amount of F508-del escaped from the ER and trafficked to the plasma membrane.

In order to determine where these two truncation mutants are located within the cell, we labeled them with mCherry on their N-termini. Fig. 2 shows a strong co-localization between $\Delta 264$ CFTR (Fig. 2A, left panel), $\Delta 27-264$ CFTR (Fig. 2C, right panel), and KDEL, the ER marker. In contrast, there was little overlap between $\Delta 264$ CFTR (Fig. 2B, left panel), $\Delta 27$ 264 CFTR (Fig. 2D, right panel), and Golgin-97, the trans-Golgi marker protein [28], demonstrating that the trafficking of both truncated versions of CFTR is arrested mostly within the ER, from which they are efficiently degraded by the proteasome, facilitated by VCP [16]. It also shows that some trafficking of the truncated mutants does occur, allowing them to reach the Golgi. The data also show that the addition of 27 amino acids to the N-terminal in $\Delta 27-264$ CFTR, which we have previously shown to increase the steady-state level of the protein [16], results in behavior similar to that of $\triangle 264$ CFTR. Although it was primarily located within the ER, there was also some transit of the truncated protein out of the ER and detectable colocalization with Golgin-97 within the Golgi.

\section{Co-localization occurs within the ER}

Because when present alone, $\Delta 264$ CFTR and $\Delta 27-264$ CFTR are each found within the ER, with only little detectable localization with Golgin-97, we asked whether F508-del and the truncated versions

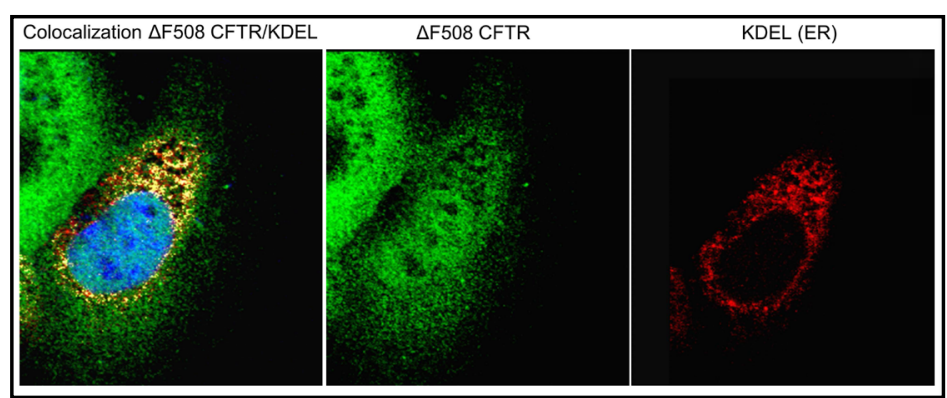

Fig. 1. F508-del co-localizes with the ER marker KDEL. CFBE410 cells stably expressing F508-del- CFBE410 cells were transfected with KDEL-td-tomato plasmid (Red, ER) and plated on coverslips. After $48 \mathrm{~h}$, the cells were fixed, permeabilized, and stained with DAPI (blue, nucleus) and antibodies against CFTR (green, CFTR 596). Representative extended-focus images are shown. The cell shown is one representative example of F508-del /KDEL colocalization (yellow) taken from three independent experiments. All cells that were successfully transfected with the KDEL-td-tomato plasmid displayed co-localization (yellow) between KDEL and F508-del. The location of the green dots over the nucleus in the middle panel occurred because the level of focus of this image includes the cytoplasm, which contains F508-del and the nuclear marker (DAPI). Note, however, that in the left panel that there is no co-localization between the nuclear marker DAPI and F508-del, indicating that F508-del is not located within the nucleus. 


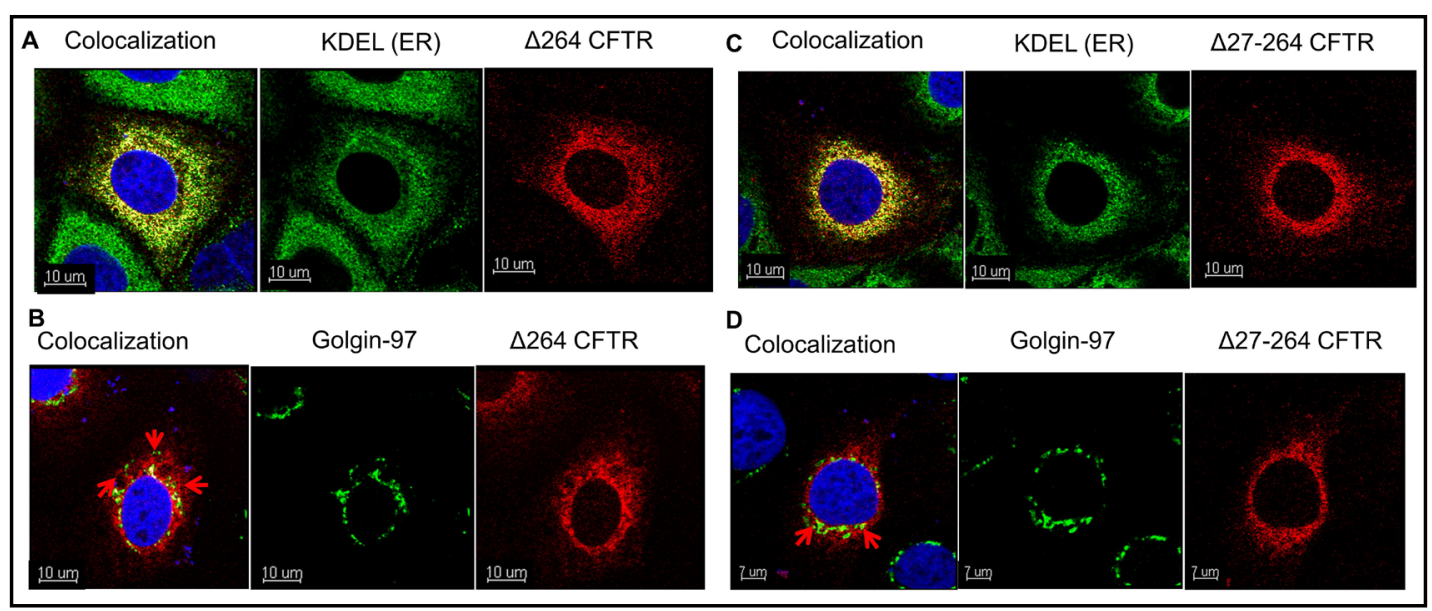

Fig. 2. $\Delta 264$ and $\Delta 27-264$ CFTR are primarily located in the ER, but some reaches the Golgi. (A) CFBE41o parental cells were plated on coverslips and transfected with $\Delta 264$ CFTR-mCherry (red, CFTR). At $48 \mathrm{~h}$ post-transfection, the cells were fixed, permeabilized, and stained with DAPI (blue, nucleus) and antibodies against KDEL (green, ER). Close association or co-localization is indicated by a yellow signal. (B) Cells were treated the same way as in (A) but stained with antibodies against Golgin-97 (green, Golgi). The red arrows indicate close association or co-localization between $\triangle 264$ CFTR and Golgin-97. (C) CFBE41o- parental cells were plated on coverslips and transfected with $\Delta 27-264$ CFTR-mCherry (red, CFTR). (D) Cells were treated the same way as in (A) but stained with antibodies against Golgin-97 (green, Golgi). The red arrows indicate close association or co-localization between $\Delta 27-264$ CFTR and golgin-97. Representative extended-focus images are shown. The data shown were selected from four independent experiments. Representative extended-focus images are shown.

of CFTR are located together within the ER, concomitant with the process of transcomplementation. For this purpose, we conducted a triple-label co-localization experiment with F508-del, $\Delta 264$ (Fig. 3A), or $\Delta 27-264$ (Fig. 3B) and the ER marker, KDEL. Fig. 3 shows the co-localization between $\Delta 264$ and $\Delta 27-264$ CFTR, respectively, F508-del and KDEL, demonstrating that co-localization of the endogenous F508-del and the truncated versions of CFTR occurs within the ER to form a transcomplementing complex consisting of F508-del and either $\Delta 264$ or $\Delta 27-264$ CFTR. The question, then, is whether the transcomplementing complex moves out of the ER. To begin to address this question, we conducted a triplelabel co-localization experiment with F508-del, $\Delta 264$ (Fig. 4A), or $\Delta 27-264$ (Fig. 4B) and the trans-Golgi marker, Golgin 97. Fig. 4 shows that there is readily detectable co-localization of F508-del and the transcomplementing truncation mutants within the trans-Golgi. Next, we determined whether a complex of proteins reaches the plasma membrane. For this purpose we conducted a triple-label co-localization experiment with F508-del, $\Delta 264$ (Fig. 5A), or $\Delta 27-264$ (Fig. 5B) and the plasma membrane marker, E-cadherin [29]. Fig. 5 shows that F508-del and the transcomplementing truncation mutants, $\Delta 264$ or $\Delta 27-264$, do indeed colocalize at the plasma membrane.

Association of F508-del with the transcomplementing truncation mutants occurs within the ER

To test whether the transcomplementing truncated CFTR and F508-del are associated within the ER, we utilized an acceptor-photobleaching FRET assay. We measured FRET by photobleaching within the perinuclear region of co-transfected CFBE410- cells. Fig. 6 represents experiments in which F508-del and $\Delta 264$ CFTR (A) or $\Delta 27-264$ CFTR (B) were cotransfected. Significant FRET was detected in both experiments. Given that Förster resonance energy transfer [30] is dependent upon the sixth power of the distance between the fluorophores, the distance between F508-del and 264 CFTR or $\Delta 27-264$ CFTR must be between 1 and $10 \mathrm{nM}$ to attain significant FRET between them. The confocal microscopy data presented 


\section{Cellular Physiology and Biochemistry
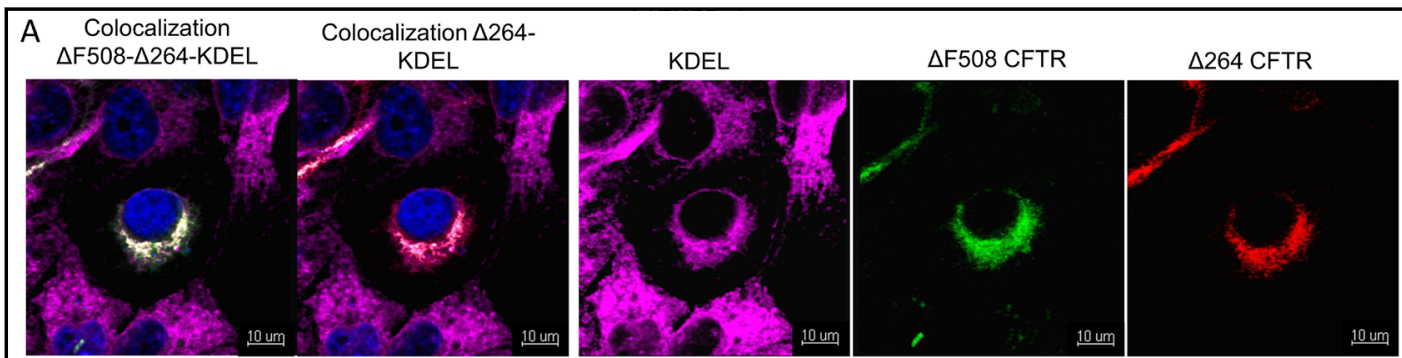

B Colocalization $\triangle$ F508- $\triangle 27-264-K D E L$

Colocalization $\Delta 27-264$ KDEL

KDEL

$\triangle F 508$ CFTR

$\triangle 27-264$ CFTR
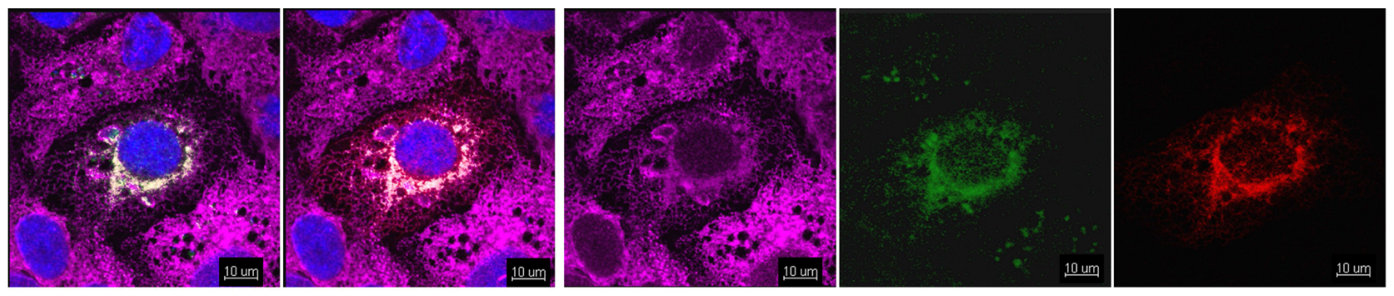

Fig. 3. (A) $\triangle 264$ CFTR and F508-del are localized to the ER. CFBE410- parental cells were plated on coverslips and co-transfected with $\triangle 264$ CFTR-mCherry (red, $\Delta 264$ CFTR) and F508-del CFTR-GFP (green, F508-del). At $48 \mathrm{~h}$ post-transfection, the cells were fixed, permeabilized, and stained with DAPI (blue, nucleus) and antibodies against KDEL (purple, ER). Close association or co-localization is indicated by a yellow signal. Cotransfection of F508-del CFTR-GFP and $\Delta 264$ CFTR-mCherry occurred in $21.1 \%$ of the cells $(27 / 128)$; of the cells stained for KDEL (purple), 100\% (11/11) showed co-localization or close association between $\Delta 264$ CFTR and F508-del. (B) Localization of $\triangle 27-264$ CFTR and F508-DEL CFTR in the ER. CFBE410 parental cells were plated on coverslips and co-transfected with $\Delta 27-264$ CFTR-mCherry (red, $\Delta 27-264$ CFTR) and F508-des CFTR-GFP (green, F508-del). Co-transfection of F508-DEL CFTR-GFP and $\Delta 27-264$ CFTR-mCherry occurred in 23.5\% of the cells (35/150); of the cells stained for KDEL (purple), 100\% (15/15) showed co-localization or close association between $\Delta 27-264$ CFTR and F508-del Representative extended-focus images are shown, and data were selected from one of four independent experiments.

above and the FRET data presented here are consistent with transcomplementation occurring by a direct interaction between F508-del and the transcomplementing truncated forms. The net effect is to restore the stability of F508-del.

\section{Transcomplementation has functional consequences}

For transcomplementation to be a useful therapy for CF, there must be enough rescue of function to have clinical benefit. We therefore measured Isc; as a first step, we exposed CFBE41o- cells stably expressing wt CFTR to AAV containing $\triangle 264$ CFTR or $\Delta 27-264$ CFTR. We have shown previously that steady-state levels of wt-CFTR are increased following transcomplementation by AAV containing $\Delta 264$ CFTR or $\Delta 27-264$ CFTR[15]. Fig. 7A shows that this treatment produced an approximately 2 -fold increase in wt-CFTR-generated currents. To evaluate the effectiveness of transcomplementation, we exposed cells in which F508-del was stably expressed at well above endogenous levels (Fig. 7B) or parental cells (CFBE410) (Fig. 7C) that express only endogenous levels of F508-del to AAV containing either $\Delta 264$ or $\triangle 27-264$ CFTR. Although the magnitude of the currents was lower in the parental CFBE cells when compared to the cells expressing F508-del, the 2-fold increase in Isc was the same in both cases. 


\section{Cellular Physiology and Biochemistry

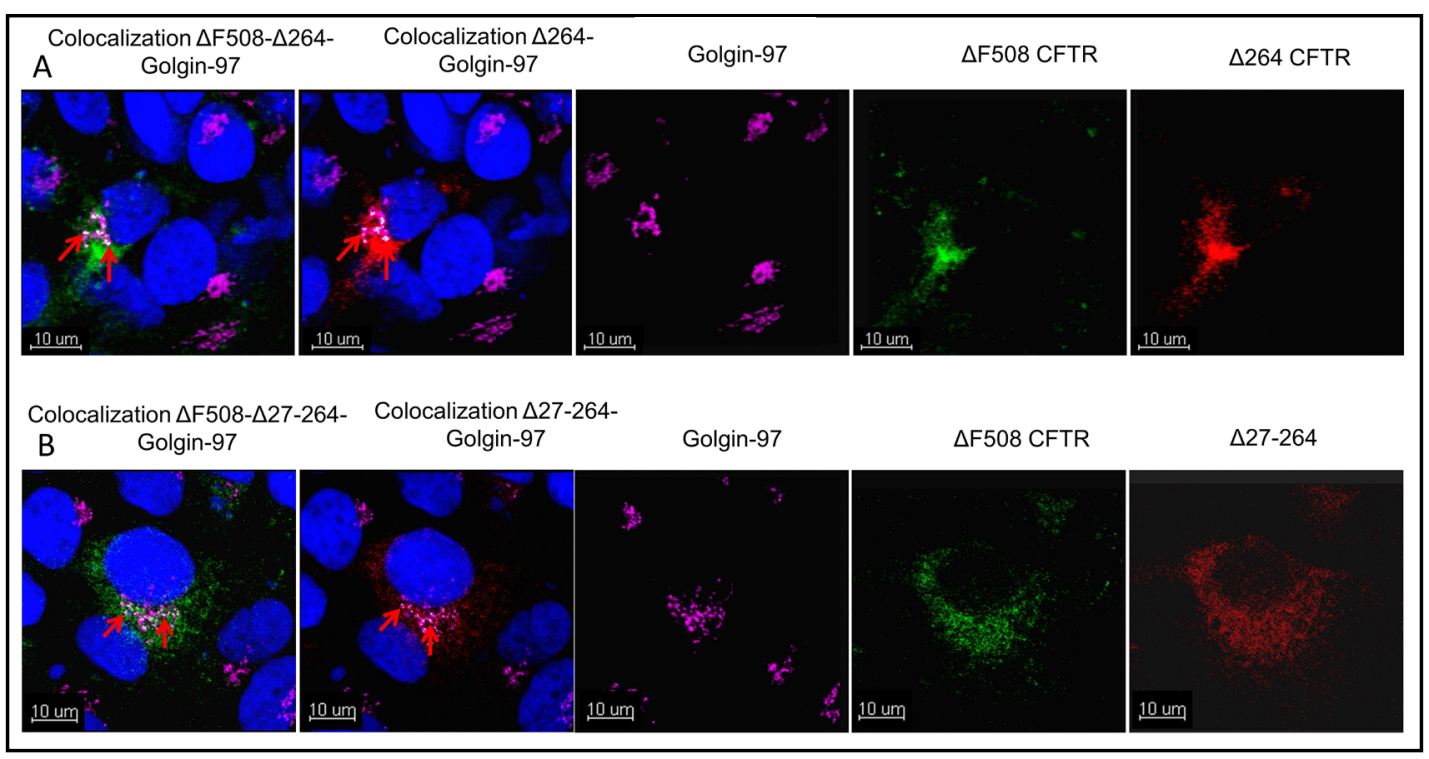

Fig. 4. (A) Co-transfection of $\triangle 264$ CFTR and F508-del results in Golgi localization. CFBE41o- parental cells were plated on coverslips and co-transfected with $\Delta 264$ CFTR-mCherry (red, $\Delta 264$ CFTR) and F508-des CFTR-GFP (green, F508-del). At $48 \mathrm{~h}$ post-transfection, the cells were fixed, permeabilized, and stained with DAPI (blue, nucleus) and antibodies against golgin-97 (purple, Golgi). The yellow signal with red arrows indicates close association or co-localization between $\Delta 264$ CFTR and Golgin-97. Representative extendedfocus images are shown, and data were selected from one of four independent experiments. Co-transfection of F508-des CFTR-GFP and $\Delta 27-264$ CFTR-mCherry occurred in 21.1\% of the cells (27/128); of the cells stained for golgin-97 (purple), 100\% (6/6) showed co-localization or close association between $\Delta 264$ CFTR and F508-del. (B) Co-transfection of $\Delta 27-264$ CFTR and F508-del results in Golgi localization. CFBE410parental cells were plated on coverslips and co-transfected with $\Delta 27-264$ CFTR-mCherry (red, $\Delta 27-264$ CFTR) and F508-del CFTR-GFP (green, F508-del). Co-transfection of F508-DEL CFTR-GFP and $\Delta 27-264$ CFTR-mCherry occurred in 23.5\% of the cells (35/150); of the cells stained for golgin-97 (purple), 100\% (11/11) showed co-localization or close association between $\Delta 27-264$ CFTR and F508-del. Representative extended-focus images are shown, and data were selected from one of four independent experiments.

\section{Discussion}

Misfolded proteins in CF are the result of several mutations in the CF gene [3]. The F508-del mutation is the most common mutation, and it causes at least two major problems with the protein without resulting in major changes in the structure of the NBD1 [31, 32]. It is now well known that the mutation destabilizes the NBD1 domain at physiological temperatures and disrupts the interaction of the NBD1 domain with the transmembrane domains [32]. Possible mechanisms to rescue these defects have received intense research interest. We and others have shown that F508-del in NBD1 and other trafficking mutants in NBD2 can be rescued by transcomplementation [33]. The major question is how the transcomplementation rescues F508-del. Here we show that $\Delta 264$ CFTR and $\Delta 27-264$ CFTR, when transfected individually into cells, are localized primarily to the ER and to some extent within the Golgi. This localization is consistent with their recognition by ERAD and degradation in proteasomes [16]. Interestingly, when they are co-expressed with F508-del, the transcomplementing mutants strongly localize with F508-del within the ER, but are then found at the trans-Golgi and the plasma membrane. These results, taken together, lead us to hypothesize that transcomplementation occurs with the ER and that the truncation mutants and F508-del move to the plasma membrane.

The FRET between $\Delta 264$ CFTR or $\Delta 27-264$ CFTR and F508-del suggests that transcomplementation takes place when the two molecules are in very close proximity. These results, 


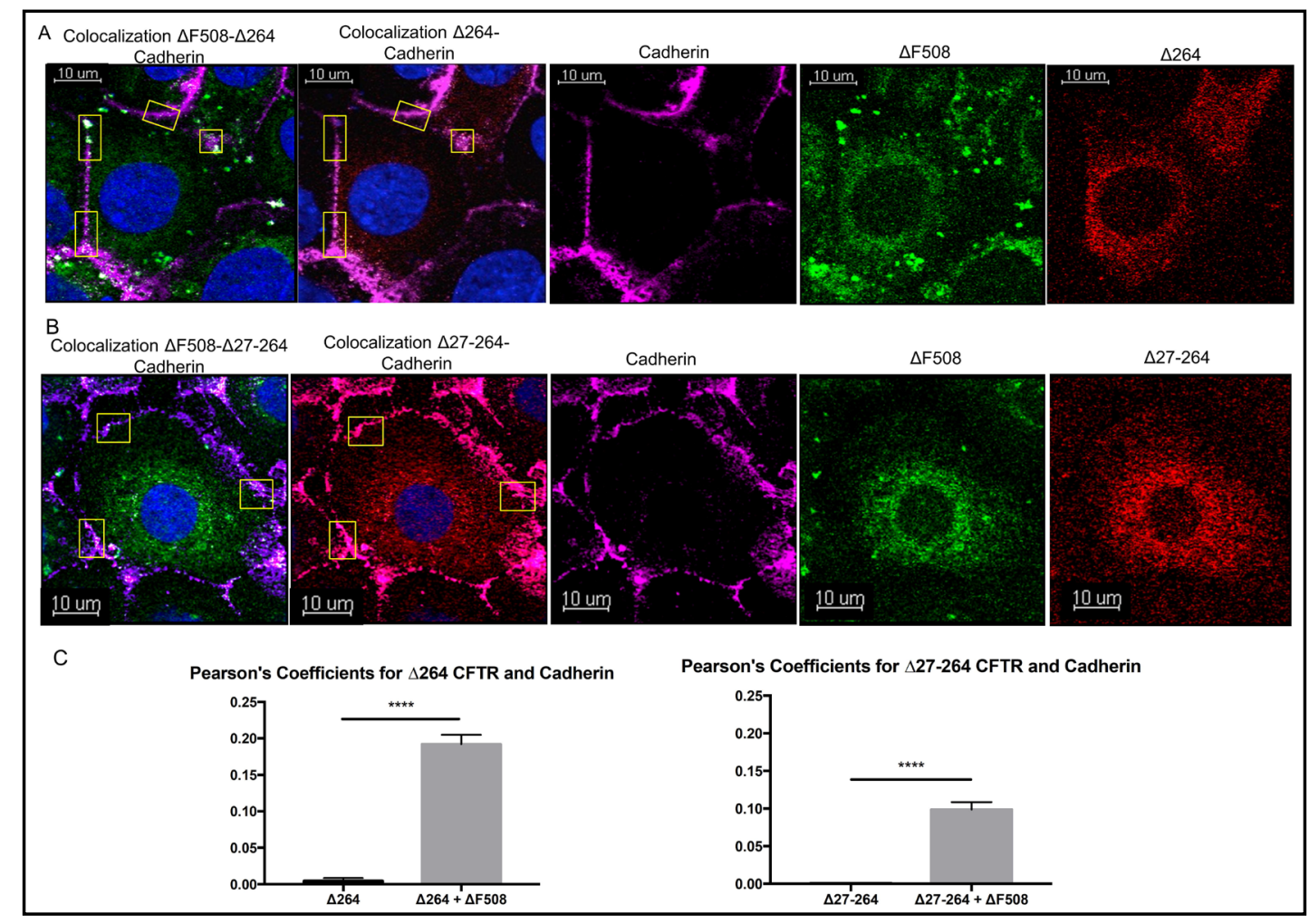

Fig. 5. (A) $\triangle 264$ CFTR and F508-del become localized to the plasma membrane. (A) CFBE410 parental cells were plated on coverslips and co-transfected with $\Delta 264$ CFTR-mCherry (red, $\Delta 264$ CFTR) and F508del CFTR-GFP (green, F508-del). At $48 \mathrm{~h}$ post-transfection, the cells were fixed, permeabilized, and stained with DAPI (blue, nucleus) and antibodies against cadherin (purple, plasma membrane). The yellow boxes indicate areas where there is close association or co-localization between $\Delta 264$ CFTR and cadherin. Cotransfection of F508-del CFTR-GFP and $\Delta 264$ CFTR-mCherry occurred in $21.1 \%$ of the cells $(27 / 128)$; of the cells stained for cadherin (purple), 100\% (10/10) showed co-localization or close association between $\Delta 264$ CFTR and F508-del. (B) $\Delta 27-264$ CFTR and F508-del become localized to the plasma membrane. (A) CFBE410- parental cells were plated on coverslips and co-transfected with $\Delta 27-264$ CFTR-mCherry (red, $\Delta$ 27-264 CFTR) and F508-del CFTR-GFP (green, F508-del). Co-transfection of F508-DEL CFTR-GFP and $\Delta 27-264$ CFTR-mCherry occurred in $23.5 \%$ of the cells (35/150); of the cells stained for cadherin (purple), 100\% (9/9) showed co-localization or close association between $\Delta$ 27-264 CFTR and F508-del. Representative extended-focus images are shown, and data were selected from one of four independent experiments. (C) The graphs show Pearson's correlation coefficients of $\Delta 264$ CFTR and $\Delta 27-264$ CFTR and cadherin when F508-del CFTR is co-transfected into cells, as compared to control samples (no F508-del CFTR). The coefficients were calculated using Imaris Imaging Software. Pearson's correlation coefficients were increased with F508-del CFTR co-transfection. Results are means \pm SE. $(n=4) * * * * p<0.0001$.

together with earlier binding studies [15], lead us to suggest that transcomplementation occurs via a bimolecular interaction between the transcomplementing truncated form and F508-del. We showed previously that when transcomplementation occurs, F508-del is functionally repaired and its residence time at the plasma membrane is extended $[15,33]$. Several studies have demonstrated transcomplementation of F508-del utilizing different truncated forms of CFTR [33]. What they all have in common is the NBD1 domain. We would hypothesize that the most likely scenario is that the transcomplementing truncated CFTR acts as a molecular chaperone [15] by binding to F508-del and allowing it to attain a stable and functional conformation that allows it to traffic to and function normally at the plasma membrane. 


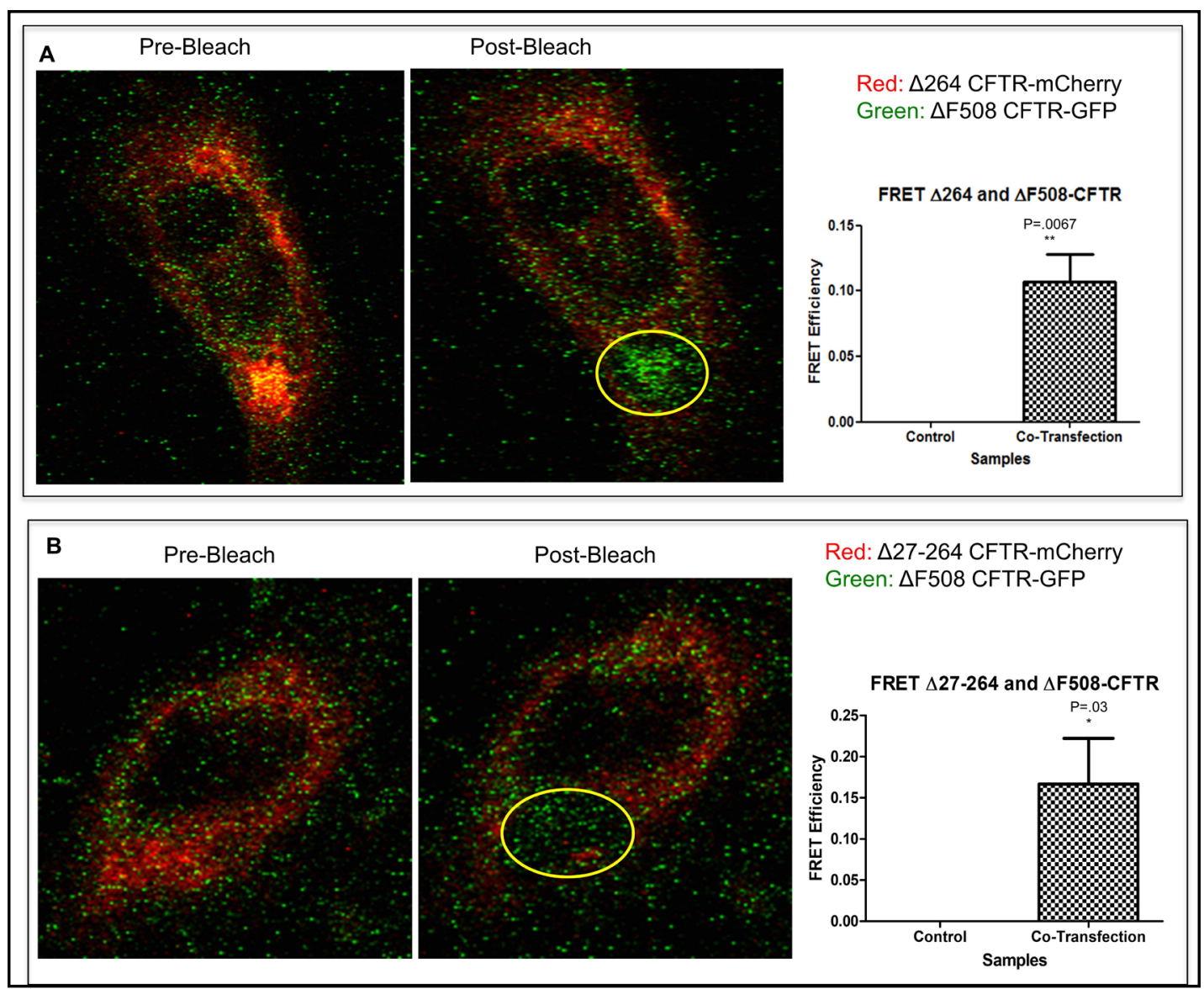

Fig. 6. $\Delta 264$ and $\Delta 27-264$ CFTR interact with F508-del. CFBE41o-cells were plated on coverslips and transfected with $\Delta 264$ (A) or $\Delta 27-264$ CFTR-mCherry (B) and $\Delta$ F508 CFTR-GFP. At 48 h post-transfection, the cells were fixed, and slides were mounted using mounting medium without anti-fade. Acceptor photobleaching was performed. Samples were bleached using the $561 \mathrm{~nm}$ laser line of a Zeiss LSM 510 confocal microscope. Samples were then excited using the 488-nm laser line, and changes in green fluorescence were measured. (A) The left column is the image before photobleaching with a 561-nm laser, and the right column is the image after photobleaching. FRET efficiency was calculated by measuring the changes in the 488-nm signal before and after photobleaching. The statistical analysis of the FRET efficiency was obtained from the images. Results are means $\pm S E(n=4)$. The FRET efficiency for the control sample is 0 , since there was no green signal present to measure because of the single transfection.

Will the restoration of function by transcomplementation be therapeutic? Since therapeutic transcomplementation would have to restore the function of the endogenous F508del, a therapeutic benefit would fundamentally depend upon the efficiency of the rescue and the magnitude of the level of expression of the mutant F508-del. We addressed these requirements by utilizing CFBE410 cells, which stably express additional F508-del, and the parental cells, which only express small amounts of endogenous CFTR. Neither $\triangle 264$ CFTR nor $\Delta 27-264$ CFTR produces chloride currents on its own [15], but each can rescue functional F508-del chloride channels. Therefore, we measured the short-circuit current in each of these cell lines. Although the magnitude of the currents was greater in the cells stably expressing additional F508-del, there was a 2-fold increase in the currents generated after rescue by transcomplementation, in both the cells stably expressing additional F508-del and in parental cells expressing endogenous levels of F508-del. Cormet-Boyaka and colleagues [25], using their transcomplementation vector, also showed rescue of CFTR-generated $\mathrm{Cl}^{-}$ currents in F508-del- containing CFBE410 cells. In addition, they observed that CFTR-dependent $\mathrm{Cl}^{-}$transport was rescued in F508-del-bearing CF mice, as assessed by nasal po- 


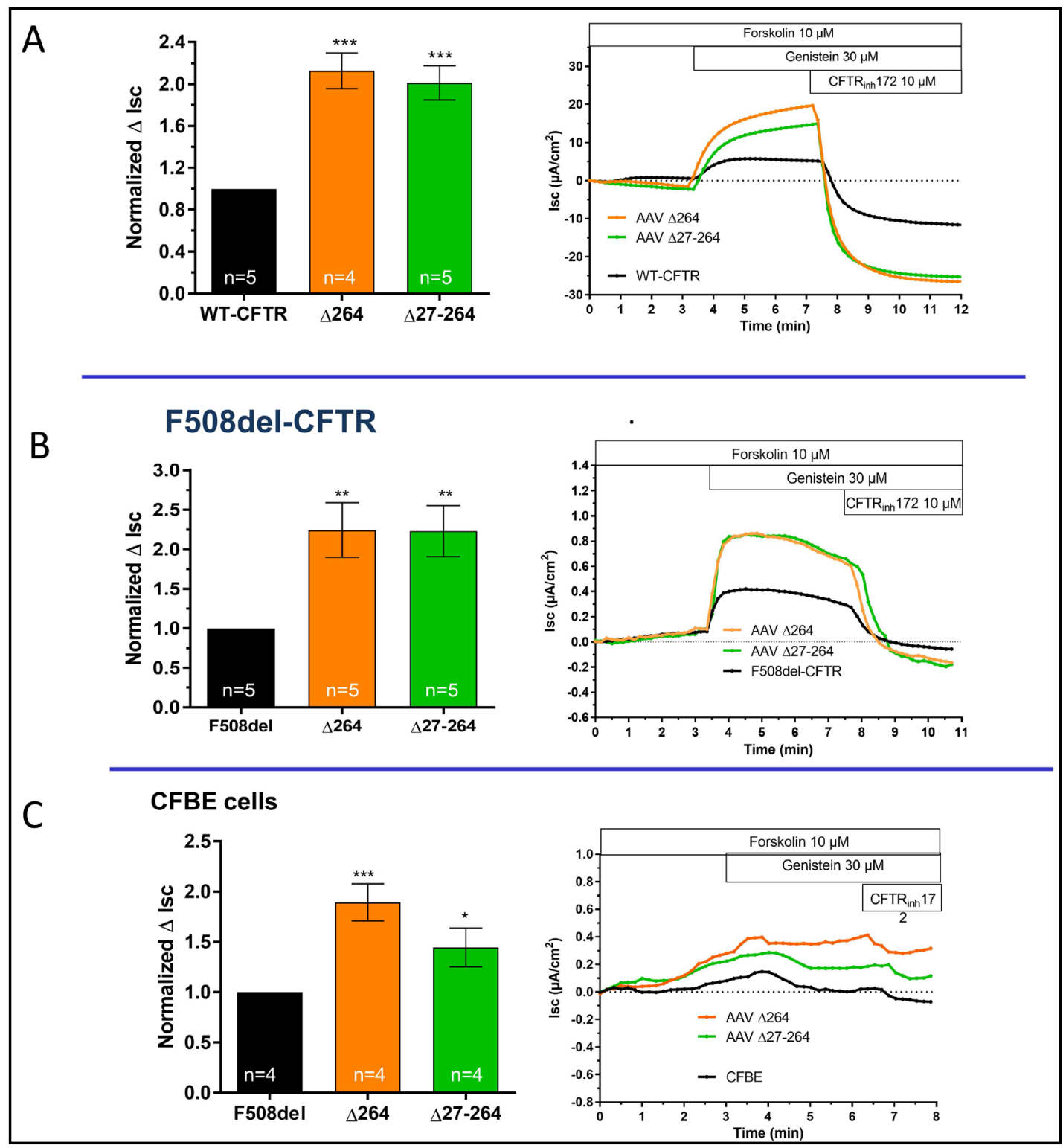

Fig. 7. Functional restoration of F508-del. Ussing chamber experiments were performed to evaluate CFTR-mediated $\mathrm{Cl}^{-}$secretion in parental CFBE410- cells or cells stably expressing F508-del (F508del) or wt CFTR after treatment with either $\Delta 27-264$ - or $\Delta 264$-containing AAV2/1 virus. The data show that transcomplementation rescues the function of wt CFTR (A), F508-del (B) and parental cells (C). Original short-circuit current recordings in untreated cells (black line) or after incubation of $\Delta 27-264-$, or $\Delta 264$ -containing AAV2/1 virus (10 $\mu \mathrm{l}, 7$ days) in cells kept at $37^{\circ} \mathrm{C}$. Corresponding $\Delta \mathrm{Isc}$ summary data are normalized to untreated CFBE410 cells. Data are expressed as the $\mathrm{CFTR}_{\text {inh }} 172$-sensitive short-circuit current $(\Delta \mathrm{Isc})$, calculated by subtracting the Isc after $\mathrm{CFTR}_{\mathrm{inh}} 172$ treatment from the peak forskolingenistein-stimulated Isc. Normalized responses calculated by normalizing the $\Delta$ Isc response to the untreated condition. Statistical significance is indicated as follows: ns, no significant difference; ${ }^{*} \mathrm{P}<0.05$; ${ }^{* *} \mathrm{P}<0.01$; ${ }^{* * *} \mathrm{P}<0.001$ ( $\mathrm{n}=4-5$ for each condition) when compared with the control condition. Amiloride $(100 \mu \mathrm{M})$ was present during the whole duration of each experiment to avoid interference by ENaC-mediated $\mathrm{Na}^{+}$currents.

tential difference, when their transcomplementing vector was instilled into the nose using an adenoviral vector. These data suggest that transcomplementation will be therapeutic; however, definitive proof of this concept awaits further study. 


\section{Cellular Physiology Cell Physiol Biochem 2019;52:1267-1279

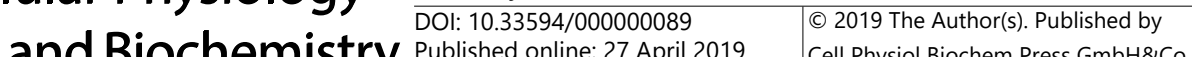 and Biochemistry Published online: 27 April 2019 Cell Physiol Biochem Press GmbH\&Co. KG

For patients with CF, new therapies based on chemical compounds that either potentiate chloride channel activity or rescue the processing of mutant CFTR have been approved by the FDA. The most striking effect of these therapies was first observed in the treatment of patients with G551D using the potentiator Ivacaftor (VX-770) [34, 35]. Subsequently, Orkambi, the combination of the potentiator Kalydeco (Ivacaftor) and the corrector Lumacaftor (VX-809), was approved for the treatment of patients with homozygous F508-del mutations [36]. In targeting the basic defects caused by certain mutations in the gene encoding CFTR, these new therapies provide a substantial improvement over previous treatments, which mainly targeted the symptoms associated with this progressive disease. However, there are over 1000 different mutations in CF patients [3], and many of these mutations affect CFTR in ways that are either too severe to be treated by VX-770, VX-809, or their combination, or that cannot be rescued at all by the existing therapies. Therefore, the most fundamental way to help every patient with CF is with gene therapy. As in any gene therapeutic approach for $\mathrm{CF}$, there are numerous challenges before an efficient clinical therapy can be achieved. These challenges include targeting the proper cells necessary for restoration of CFTR function, achieving therapeutic levels of gene transfer and transduction or endogenous gene editing, and overcoming the body's defenses against the introduction of foreign DNA, which may include both innate and acquired immunity (reviewed in [5, 37]). However, given the renewed enthusiasm for gene therapeutic approaches, this is an opportune time for overcoming these challenges.

\section{Acknowledgements}

The authors wish to thank Dr. Deborah McClellan for editing the manuscript. The research was funding by the Cystic Fibrosis Foundation GUGGIN14XX0 and an NHLBI multi-PI grant, R01 HL122267, to LC and WBG.

EASB, IS, CB, VC, and LC conducted experiments; WBG interpreted data and wrote the manuscript; LC designed and conducted the studies, interpreted data, and wrote the manuscript.

\section{Disclosure Statement}

None of the authors has any financial interests that pertain directly to this work.

\section{References}

1 Fuller CM, Benos DJ: Cftr! Am J Physiol 1992;263:C267-286.

- 2 Riordan JR: Cystic fibrosis as a disease of misprocessing of the cystic fibrosis transmembrane conductance regulator glycoprotein. Am J Hum Genet 1999;64:1499-1504.

3 Sosnay PR, Siklosi KR, Van GF, Kaniecki K, Yu H, Sharma N, Ramalho AS, Amaral MD, Dorfman R, Zielenski

J, Masica DL, Karchin R, Millen L, Thomas PJ, Patrinos GP, Corey M, Lewis MH, Rommens JM, Castellani

C, Penland CM, et al.: Defining the disease liability of variants in the cystic fibrosis transmembrane conductance regulator gene. NatGenet 2013;45:1160-1167.

4 Welsh MJ, Smith AE: Molecular mechanisms of CFTR chloride channel dysfunction in cystic fibrosis. Cell 1993;73:1251-1254.

5 Guggino WB, Cebotaru L: Adeno-Associated Virus (AAV) gene therapy for cystic fibrosis: current barriers and recent developments. Expert Opin Biol Ther 2017;17:1265-1279.

6 Moss RB, Rodman D, Spencer LT, Aitken ML, Zeitlin PL, Waltz D, Milla C, Brody AS, Clancy JP, Ramsey B, Hamblett N, Heald AE: Repeated adeno-associated virus serotype 2 aerosol-mediated cystic fibrosis transmembrane regulator gene transfer to the lungs of patients with cystic fibrosis: a multicenter, doubleblind, placebo-controlled trial. Chest 2004;125:509-521. 


\section{Cellular Physiology Cell Physiol Biochem 2019;52:1267-1279 \begin{tabular}{l|l}
\hline DOI: 10.33594/000000089 & (c)19 The Author(s). Published by
\end{tabular} and Biochemistry Published online: 27 April $2019 \quad$ Cell Physiol Biochem Press GmbH\&Co. KG

7 Moss RB, Milla C, Colombo J, Accurso F, Zeitlin PL, Clancy JP, Spencer LT, Pilewski J, Waltz DA, Dorkin HL, Ferkol T, Pian M, Ramsey B, Carter BJ, Martin DB, Heald AE: Repeated aerosolized AAV-CFTR for treatment of cystic fibrosis: a randomized placebo-controlled phase 2B trial. Hum Gene Ther 2007;18:726-732.

8 Ding W, Zhang L, Yan Z, Engelhardt JF: Intracellular trafficking of adeno-associated viral vectors. Gene Ther 2005;12:873-880.

- 9 Flotte TR, Afione SA, Solow R, Drumm ML, Markakis D, Guggino WB, Zeitlin PL, Carter BJ: Expression of the cystic fibrosis transmembrane conductance regulator from a novel adeno-associated virus promoter. J Biol Chem 1993;268:3781-3790.

10 Gao G, Vandenberghe LH, Wilson JM: New recombinant serotypes of AAV vectors. Curr Gene Ther 2005;5:285-297.

- 11 Xu L, Daly T, Gao C, Flotte TR, Song S, Byrne BJ, Sands MS, Parker PK: CMV-beta-actin promoter directs higher expression from an adeno-associated viral vector in the liver than the cytomegalovirus or elongation factor 1 alpha promoter and results in therapeutic levels of human factor X in mice. Hum Gene Ther 2001;12:563-573.

12 Zhang LN, Karp P, Gerard CJ, Pastor E, Laux D, Munson K, Yan Z, Liu X, Godwin S, Thomas CP, Zabner J, Shi H, Caldwell CW, Peluso R, Carter B, Engelhardt JF: Dual therapeutic utility of proteasome modulating agents for pharmaco-gene therapy of the cystic fibrosis airway. Mol Ther 2004;10:990-1002.

- 13 Sirninger J, Muller C, Braag S, Tang Q, Yue H, Detrisac C, Ferkol T, Guggino WB, Flotte TR: Functional characterization of a recombinant adeno-associated virus 5-pseudotyped cystic fibrosis transmembrane conductance regulator vector. Hum Gene Ther 2004;15:832-841.

- 14 Vidović D, Carlon MS, da Cunha MF, Dekkers JF, Hollenhorst MI, Bijvelds MJ, Ramalho AS, Van den Haute C, Ferrante M, Baekelandt V: rAAV-CFTR $\Delta$ R rescues the cystic fibrosis phenotype in human intestinal organoids and cystic fibrosis mice. Am J Respir Crit Care Med 2016;193:288-298.

- 15 Cebotaru L, Woodward O, Cebotaru V, Guggino WB: Transcomplementation by a truncation mutant of cystic fibrosis transmembrane conductance regulator (CFTR) enhances DeltaF508 processing through a biomolecular interaction. J Biol Chem 2013;288:10505-10512.

16 Cebotaru L, Vij N, Ciobanu I, Wright J, Flotte T, Guggino WB: Cystic fibrosis transmembrane regulator missing the first four transmembrane segments increases wild type and DeltaF508 processing. J Biol Chem 2008;283:21926-21933.

17 Devidas S, Yue H, Guggino WB: The second half of the cystic fibrosis transmembrane conductance regulator forms a functional chloride channel. J Biol Chem 1998;273:29373-29380.

- 18 Naren AP, Quick MW, Collawn JF, Nelson DJ, Kirk KL: Syntaxin 1A inhibits CFTR chloride channels by means of domain-specific protein-protein interactions. Proc Natl Acad Sci U S A 1998;95:10972-10977.

- 19 Fischer AC, Smith CI, Cebotaru L, Zhang X, Askin FB, Wright J, Guggino SE, Adams RJ, Flotte T, Guggino WB: Expression of a truncated cystic fibrosis transmembrane conductance regulator with an AAV5pseudotyped vector in primates. Mol Ther 2007;15:756-763.

20 Cormet-Boyaka E, Jablonsky M, Naren AP, Jackson PL, Muccio DD, Kirk KL: Rescuing cystic fibrosis transmembrane conductance regulator (CFTR)-processing mutants by transcomplementation. Proc Natl Acad Sci U S A 2004;101:8221-8226.

21 Sun F, Mi Z, Condliffe SB, Bertrand CA, Gong X, Lu X, Zhang R, Latoche JD, Pilewski JM, Robbins PD, Frizzell RA: Chaperone displacement from mutant cystic fibrosis transmembrane conductance regulator restores its function in human airway epithelia. FASEB J 2008;22:3255-3263.

22 Illek B, Maurisse R, Wahler L, Kunzelmann K, Fischer H, Gruenert DC: Cl transport in complemented CF bronchial epithelial cells correlates with CFTR mRNA expression levels. Cell Physiol Biochem 2008;22:5768.

23 Cui L, Aleksandrov L, Chang XB, Hou YX, He L, Hegedus T, Gentzsch M, Aleksandrov A, Balch WE, Riordan JR: Domain interdependence in the biosynthetic assembly of CFTR. J Mol Biol 2007;365:981-994.

24 Lopes-Pacheco M, Boinot C, Sabirzhanova I, Morales MM, Guggino WB, Cebotaru L: Combination of Correctors Rescue $\Delta$ F508-CFTR by Reducing Its Association with Hsp40 and Hsp27. J Biol Chem 2015;290:25636-25645.

25 Cormet-Boyaka E, Hong JS, Berdiev BK, Fortenberry JA, Rennolds J, Clancy JP, Benos DJ, Boyaka PN, Sorscher EJ: A truncated CFTR protein rescues endogenous DeltaF508-CFTR and corrects chloride transport in mice. FASEB J 2009;23:3743-3751. 


\section{Cellular Physiology Cell Physiol Biochem 2019;52:1267-1279

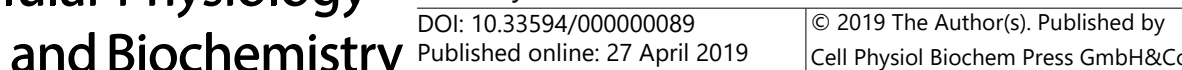 \\ \begin{tabular}{l|l} 
Published online: 27 April 2019 & Cell Physiol Biochem Press GmbH\&Co. KG \\
\hline Smith Bergbower et al : Restoring Function of F508-del by Transcomplementation
\end{tabular}}

26 Tang BL, Wong SH, Low SH, Hong W: Retention of a type II surface membrane protein in the endoplasmic reticulum by the Lys-Asp-Glu-Leu sequence. J Biol Chem 1992;267:7072-7076.

- 27 Lukacs GL, Mohamed A, Kartner N, Chang XB, Riordan JR, Grinstein S: Conformational maturation of CFTR but not its mutant counterpart (DeltaF508) occurs in the endoplasmic reticulum and requires ATP. EMBO J 1994;13:6076-6086.

28 Barr FA, Short B: Golgins in the structure and dynamics of the Golgi apparatus. Curr Opin Cell Biol 2003;15:405-413.

29 Brüser L, Bogdan S: Adherens Junctions on the Move-Membrane Trafficking of E-Cadherin. Cold Spring Harb Perspect Biol 2017:9:pii:a029140.

30 Förster T: Zwischenmolekulare Energiewanderung und Fluoreszenz. Annalen der Physik 1948;437:55-75.

- 31 Lewis HA, Zhao X, Wang C, Sauder JM, Rooney I, Noland BW, Lorimer D, Kearins MC, Conners K, Condon B, Maloney PC, Guggino WB, Hunt JF, Emtage S: Impact of the deltaF508 mutation in first nucleotide-binding domain of human cystic fibrosis transmembrane conductance regulator on domain folding and structure. J Biol Chem 2005;280:1346-1353.

32 Mendoza JL, Schmidt A, Li Q, Nuvaga E, Barrett T, Bridges RJ, Feranchak AP, Brautigam CA, Thomas PJ: Requirements for efficient correction of DeltaF508 CFTR revealed by analyses of evolved sequences. Cell 2012;148:164-174.

33 Cebotaru L, Guggino WB: Complement yourself: Transcomplementation rescues partially folded mutant proteins. Biophys Rev 2014;6:169-180.

34 Boyle MP, Bell SC, Konstan MW, McColley SA, Rowe SM, Rietschel E, Huang X, Waltz D, Patel NR, Rodman D: A CFTR corrector (lumacaftor) and a CFTR potentiator (ivacaftor) for treatment of patients with cystic fibrosis who have a phe508del CFTR mutation: a phase 2 randomised controlled trial. Lancet Respir Med 2014;2:527-538.

35 Accurso FJ, Rowe SM, Clancy JP, Boyle MP, Dunitz JM, Durie PR, Sagel SD, Hornick DB, Konstan MW, Donaldson SH, Moss RB, Pilewski JM, Rubenstein RC, Uluer AZ, Aitken ML, Freedman SD, Rose LM, MayerHamblett N, Dong Q, Zha J, et al.: Effect of VX-770 in persons with cystic fibrosis and the G551D-CFTR mutation. N Engl J Med 2010;363:1991-2003.

36 Mayer M: Lumacaftor-ivacaftor (Orkambi) for cystic fibrosis: behind the 'breakthrough'. Evid Based Med 2016;21:83-86.

37 Mueller C, Flotte TR: Gene therapy for cystic fibrosis. Clin Rev Allergy Immunol 2008;35:164-178. 